\title{
Sensitivity of upper atmospheric emissions calculations to solar/stellar UV flux
}

\author{
Mathieu Barthelemy ${ }^{1, *}$ and Gaël Cessateur ${ }^{2}$ \\ 1 UJF-Grenoble 1/CNRS-INSU, Institut de Planètologie et d'Astrophysique de Grenoble (IPAG), UMR 5274, \\ 38041 Grenoble, France \\ *Corresponding author: mathieu.barthelemy@obs.ujf-grenoble.fr \\ 2 Physical-Meteorological Observatory/World Radiation Center, Davos, Switzerland
}

Received 19 September 2014 / Accepted 20 October 2014

\begin{abstract}
The solar UV (UltraViolet) flux, especially the EUV (Extreme UltraViolet) and FUV (Far UltraViolet) components, is one of the main energetic inputs for planetary upper atmospheres. It drives various processes such as ionization, or dissociation which give rise to upper atmospheric emissions, especially in the UV and visible. These emissions are one of the main ways to investigate the upper atmospheres of planets. However, the uncertainties in the flux measurement or modeling can lead to biased estimates of fundamental atmospheric parameters, such as concentrations or temperatures in the atmospheres. We explore the various problems that can be identified regarding the uncertainties in solar/stellar UV flux by considering three examples. The worst case appears when the solar reflection component is dominant in the recorded spectrum as is seen for outer solar system measurements from HST (Hubble Space Telescope). We also show that the estimation of some particular line parameters (intensity and shape), especially Lyman $\alpha$, is crucial, and that both total intensity and line profile are useful. In the case of exoplanets, the problem is quite critical since the UV flux of their parent stars is often very poorly known.
\end{abstract}

Key words. space weather - solar spectrum (UV) - aeronomy - airglow

\section{Introduction and motivations}

Solar irradiance in the UV (UltraViolet) range is a key parameter for space climate studies (Lilensten et al. 2008; Mikhailov et al. 2012). Nowcasting the solar UV variability is extremely important for numerous applications as we shall see throughout this paper. The solar UV flux is indeed the main driver of upper atmospheres dynamics and chemistry, along with the magnetospheric energetic input, leading to ionization, excitation, and chemical processes.

In solar physics, the most energetic part of the solar UV spectrum is conventionally divided into middle UV (MUV, 200-300 nm), far UV (FUV, 122-200 nm), extreme UV (EUV, 10-121 nm), and soft X-rays (XUV, $0.1-10 \mathrm{~nm}$; Lilensten et al. 2008). The solar spectral variability in the UV is dynamic, and affects the thermosphere/ionosphere system differently on various time scales. The top panel of Figure 1 displays the solar spectrum. The bottom panel displays its variability, computed over the 11-year solar cycle, as follows:

$$
v=\left(I_{\max }(\lambda)-I_{\min }(\lambda)\right) / F_{\text {mean }}(\lambda) .
$$

Long-term variations, such as the solar magnetic cycle modulation, have a more marked impact on the shorter wavelengths, especially in the XUV and EUV ranges where the intrinsic variability can reach $100 \%-1000 \%$ as shown in Figure 1 . The variability on a 27 -day solar rotation time scale is mostly related to the appearance and disappearance of active regions at the solar surface. The center-to-limb variation also causes a 13.5-day modulation, with an excess of emission for spectral lines that exhibit limb brightening (e.g., coronal lines) and a deficit for wavelengths that exhibit limb darkening, such as the $168-210 \mathrm{~nm}$ range (Crane et al. 2004).

However, the long-term monitoring of the UV irradiance is a major challenge. Measurements must be carried out in space, where instruments suffer from aging, degradation, and signal contamination particularly severe in the EUV and FUV spectral range (BenMoussa et al. 2013). The first observational missions began in the early 1960s with some sporadic measurements, but it is only recently that continuous monitoring of the complete UV spectrum is available with the launch of two satellites: TIMED in 2002 for the EUV spectral range (Woods et al. 2005) and SORCE for the FUV and MUV parts (Rottman 2005). The data in the 27-115 nm range come from the EUV Grating Spectrograph (EGS), which is part of the Solar Extreme Ultraviolet Experiment (SEE) on board TIMED (Thermosphere Ionosphere Mesosphere Energetics and Dynamics). The instrument has a spectral resolution of $0.4 \mathrm{~nm}$, but has been rebinned to $1 \mathrm{~nm}$ for the present study. The $115-250 \mathrm{~nm}$ range is covered by the SOLar Stellar Irradiance Comparison Experiment (SOLSTICE) instrument on board the SOLar Radiation and Climate Experiment (SORCE), which also has a binsize of $1 \mathrm{~nm}$. Finally, the data in the 1-27 nm range come from the XUV Photometer System (XPS) instrument on board SORCE, which is not a spectrometer but a radiometer using several broad bandpasses. The XUV data are then processed with an algorithm using the CHIANTI spectra models (Woods et al. 2008). Although ideal, this approach relies on good calibration of the 

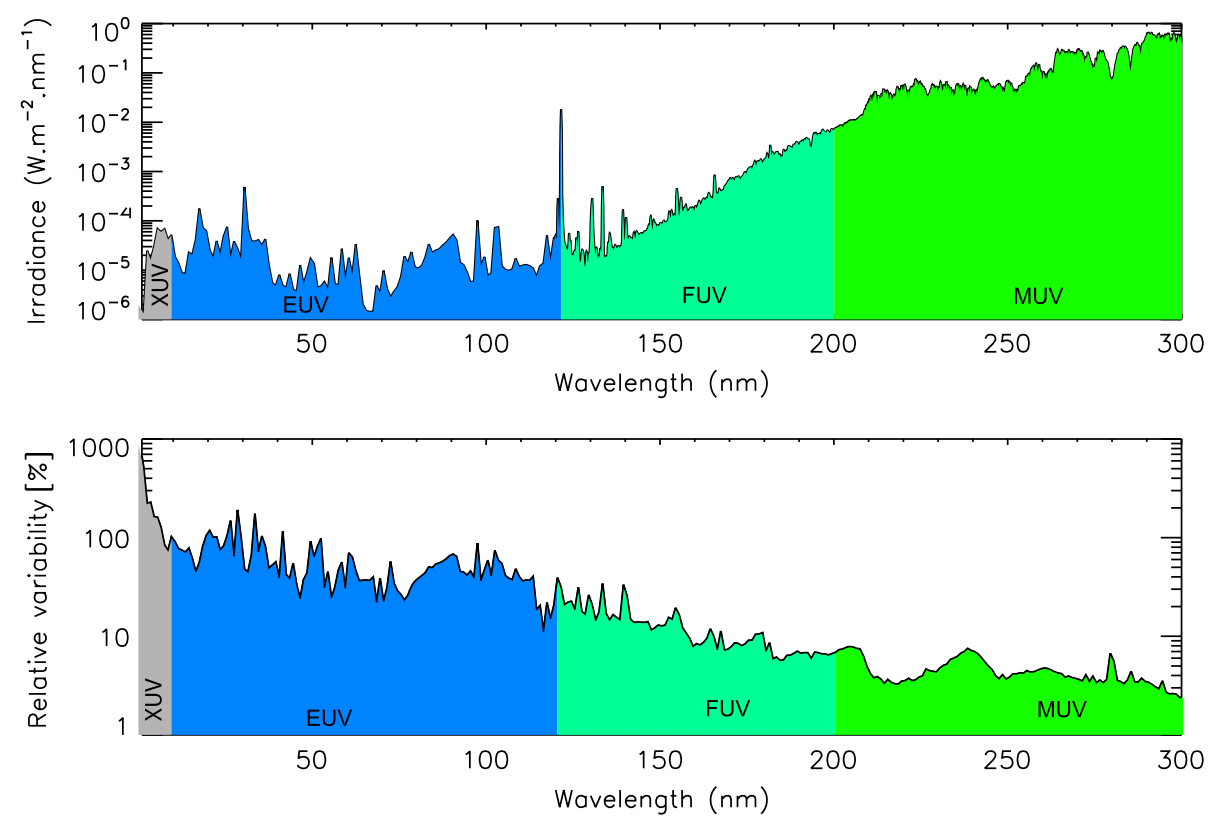

Fig. 1. Upper Panel: the solar spectrum as observed. Lower Panel: relative variability of the solar spectrum for the UV spectral range over the 11-year magnetic cycle. Adapted from Cessateur (2011).

instruments, which are largely debated in the solar community as discussed by these two papers (Haigh et al. 2010; Lean et al. 2011).

Direct UV measurements are not always available during certain epochs. Several empirical approaches for estimating a reconstruction of the solar UV variability have been developed, and planetary modelers often rely on such approaches. They either use some simple UV models based on old reference spectra from cycles 21 or 22 , while we are currently in the 24th cycle. An interpolation is performed using different solar proxies (such as the radiometric index F10.7 or the magnesium index $\mathrm{Mg}$ II) to infer the solar irradiance at a specific date. Linear combinations of these proxies and their 81-day running mean, or nonlinear functions of them (such as the E10.7 proxy) are today used in many models (Hinteregger 1981; Richards et al. 1994; Tobiska et al. 2000; Lean et al. 2003). In the following, we will use the HEUVAC model as described by Richards et al. (2006) as an empirical model reference for comparing with others solar spectra in the following.

Dudok de Wit et al. (2009) have shown that most of the solar proxies do not capture the salient features of the solar UV variability, which leads then to the development of other approaches. A physical approach consists in considering that the solar spectrum variability in the UV can be described by only a few physical terms. The variability of the solar spectrum is indeed coherent. One particular manifestation is the similar time evolution of the irradiance as observed at different wavelengths. Solar emissions coming from different solar regions across the solar atmosphere (upper photosphere, chromosphere, transition region, and lower corona) are very well correlated on time scales greater than the dynamic time of transient events such as flares (Floyd et al. 2005). This high correlation resides in the strong structuring of the solar atmosphere by the magnetic field (Domingo et al. 2009). Based on this remarkable property, we have developed a strategy for reconstructing the solar spectral irradiance from a measurement of a small set of correctly chosen passbands (Cessateur et al. 2011, 2012b). We will use the solar spectrum as reconstructed with this particular method in the following sections.

However, if the angle Earth-Sun-Planet becomes too large, solar proxies as estimated from terrestrial measurements or earth orbit-based measurements do not reflect at all the variability of the solar UV flux at the considered planet, which receives different UV irradiances and variability than Earth. It must be stressed that better results will always be obtained with a local solar UV flux measurement, which underlines the need for small UV radiometers on board each planetary missions.

The solar UV flux with the magnetospheric energetic inputs induces a large panel of processes such as ionization, dissociation, or excitation of the gases in the upper atmosphere. These processes induce electron production and photo excitation that can be measured remotely, and give rise to a large panel of observable quantities. This opens the possibility to diagnose the upper atmospheres of solar system objects but also, in the near future, of exoplanets (Menager et al. 2013). In most cases, except for the Earth case and space missions, information about planetary upper atmosphere is derived from remote sensing of the light emissions and thus the photoexcitation.

These diagnostics can be made through models such as the TRANS* family models, developed to study planetary ionospheres such as Earth (Lilensten \& Blelly 2002), Mars (Witasse et al. 2002, 2003; Simon et al. 2009), Venus (Gronoff et al. 2008), Titan (Lilensten et al. 2005a, 2005b; Gronoff et al. 2009a, 2009b), and Jupiter (Menager et al. 2010). The TRANS* family models are 1D kinetic models which solve the Boltzmann equation for the suprathermal electrons along a vertical (or a magnetic field line) in the atmosphere. Some modules allow us to calculate the effects of these supra-thermal electrons including ionization, dissociation, or excitations and thus light emissions. Gronoff et al. (2012) recently explored the uncertainties induced by the cross sections using this kind of model. Some other simulations have 
also been performed for example by Galand et al. (2011) for Saturn. Grodent et al. (2001) explored the jovian case but with only two streams, contrary to the family Trans* model, which is a multistream simulation (16 angles in most cases). Shematovich et al. (2008) also did similar calculations for example on Mars but using a Monte Carlo model.

All these models need the solar UV flux as an input. The solar flux is estimated by both measurements and models as we shall elaborate in the following sections. One goal of this paper is also to compare different solar UV models. However, for most cases studied in this paper, we will use the measured solar flux as a "reference spectrum". The aim of this paper is to explore the sensitivity of the models to different estimations of the solar flux, the resulting uncertainties on the atmospheric emissions and thus on the atmospheric parameters. Let us note that uncertainties due to solar flux are not always the major component compared to other sources of uncertainties. For example, some studies explore the uncertainties on the cross sections or reaction rates, which can be really important reaching, for example, $50 \%$ for the quenching of the $\mathrm{O}^{1} \mathrm{~S}$ state (Gronoff et al. 2012). However, the solar flux is one of the sources of uncertainties, and cannot be neglected in most cases. We will work on three "typical examples", and focus for each case on the specific problems caused by the imperfect knowledge of the solar flux. These examples are chosen because each reveals one specific problem. The aim is not to review all the atmospheric emission processes but to extract the main type of problems, which can be caused by a lack of knowledge of the solar UV flux. The case of Ganymede is interesting because due to the thin atmosphere, the link between the solar flux and the atmospheric emissions is as direct as possible. The transport of electrons is almost negligible for Ganymede. The case of Uranus is critical since the FUV solar reflected flux represents the dominant part of the Earth-based observations. The study of specific solar lines (especially H-Lyman $\alpha$ ) is important since it reveals the need for very high resolution for specific lines and radiative transfer simulation. In a last section, we will extend this problem to the exoplanet cases.

\section{Example using Ganymede: study of the importance of specific lines}

We will perform some simulations using different models of solar irradiance and direct measurements from SORCE and SOLSTICE which will be considered as the reference spectrum except when the line shapes are important. The considered models are: (i) the HEUVAC model, using the radiometric index F10.7 (Richards et al. 2006), and (ii) an empirical model based on the direct observation with band passes looking at specific spectral ranges as previously described.

The effects of the solar UV flux on Ganymede have recently been modeled by Cessateur et al. (2012a). Production of different excited states of the atmospheric species has been calculated in various geometries at different latitudes as well as electron production. We focused here on the role played by a specific solar line to the electron production and airglow emission. We used the same hypothesis for the calculation, i.e., the

\footnotetext{
${ }^{1}$ The $\mathrm{O}^{1} \mathrm{~S}$ state is responsible for the oxygen green line in terrestrial planets' upper atmosphere emissions by ground state atomic oxygen.
}

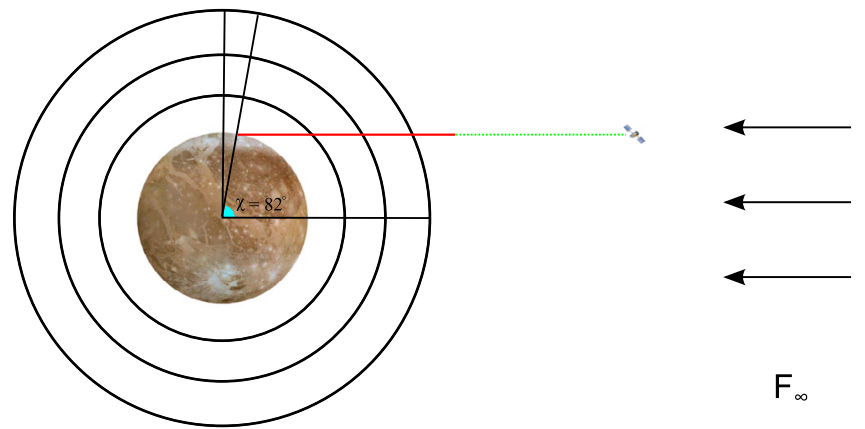

Fig. 2. Line of sight for the Ganymede case.

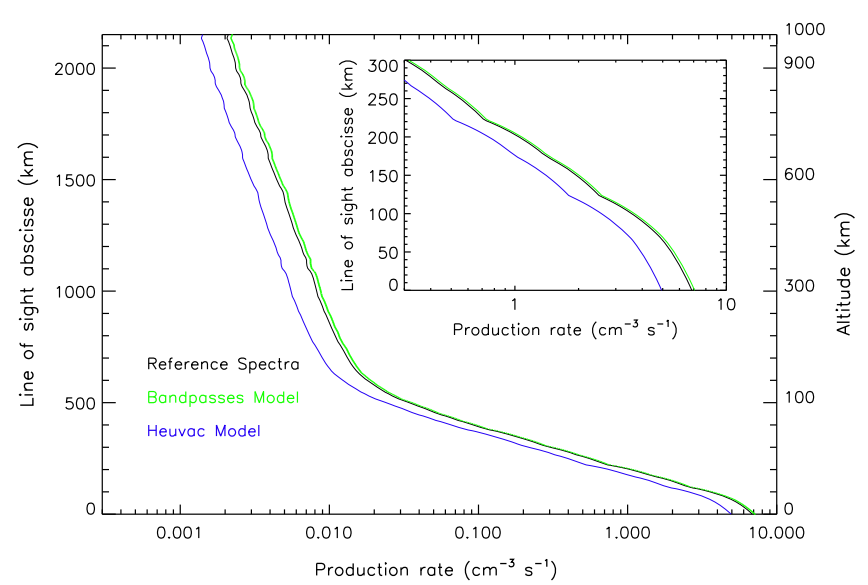

Fig. 3. Electron production rate for three different solar irradiance spectra: the reference spectrum (in black), the one obtained with a bandpass model (in green), and the HEUVAC model (in blue).

atmospheric model, built by Marconi (2007) and a line of sight parallel to the equatorial plane reaching the ground at a solar zenith angle of $82^{\circ}$ (Fig. 2). The atmosphere close to the equator is dominated by water coming from ice sublimation, while molecular oxygen dominates in the polar regions. The exobase is at the ground except near-equatorial regions where it reaches $50 \mathrm{~km}$.

\subsection{Electron production}

In the case of electron production, the part of the solar spectrum which plays an important role lies between $1 \mathrm{~nm}$ and $105 \mathrm{~nm}$. We consider here moderate solar activity, i.e., with F10.7 = 172 (November 1, 2004). Let us stress that similar conclusions regarding the disagreement between the three different solar spectra are obtained regardless of the level of solar activity.

Figure 3 displays the electron production rate within Ganymede's atmosphere. Due to its very thin atmosphere, the maximum of electronic production is close to the ground. Let us start with the HEUVAC model: the electron production at the ground reaches 5 electrons $\mathrm{cm}^{-3} \mathrm{~s}^{-1}$. This model then underestimates the electron production by about $13 \%$, while the disagreement reaches only $3 \%$ with the bandpass model. More interestingly is the evaluation of the Total Electronic Content (TEC) in Ganymede's atmosphere. Using a steadystate equilibrium model, we can evaluate the TEC by considering that the loss reaction involves only $\mathrm{O}_{2}^{+}$and $\mathrm{H}_{2}^{+}$. While the bandpass model overestimates the TEC obtained with the 


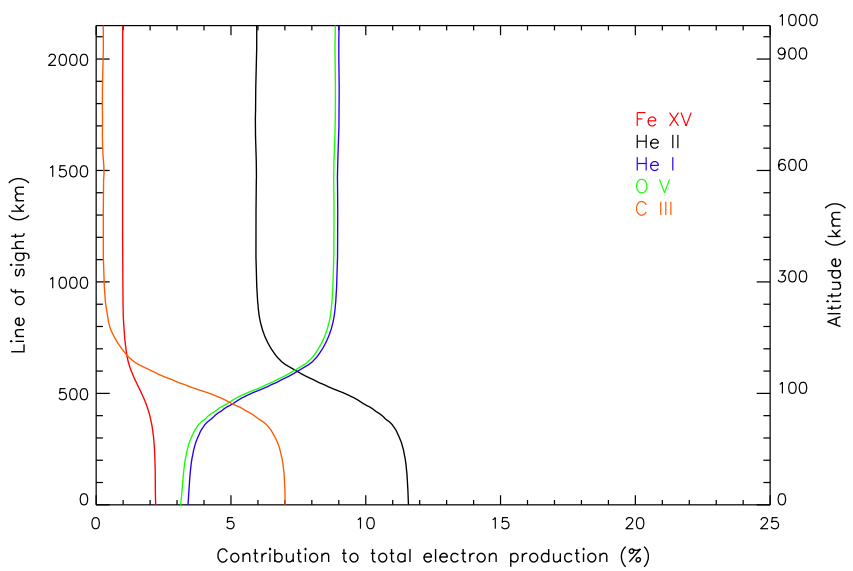

Fig. 4. Contribution to the electron production for five solar lines.

reference spectra by about $1.8 \%$, the HEUVAC model underestimates it by about $11 \%$.

For each case, a significant part of the ionization is created by a reduced number of solar lines. We considered here five solar lines, which are dominant within the solar UV flux: Fe XV $(28.4 \mathrm{~nm})$, He II $(30.4 \mathrm{~nm})$, He I $(58.4 \mathrm{~nm})$, O V $(62.5 \mathrm{~nm})$, and C III $(97.5 \mathrm{~nm})$. Those five solar lines contribute around $20 \%$ of the electron production as displayed by Figure 4. In terms of altitude, two regions can be identified with a border around $100 \mathrm{~km}$ related to the predominance of specific molecular species. At low altitudes, the He II line plays the major role, followed by the C III line representing respectively 11.5 and $7 \%$ of the total electron production on the ground. While at high altitudes the $\mathrm{O} \mathrm{V}$ and He I lines both contribute for $9 \%$ of the production, the He II line is still representing $6 \%$.

Those five lines are of strong importance in the case of Ganymede. Nevertheless in the HEUVAC model, these lines are systematically overestimated, sometimes by a factor of 2 , leading to an overestimation of the production. Considering that the global error is negative, it has to be compensated by another bias in the HEUVAC model. The continuum in the spectral region between 40 and $70 \mathrm{~nm}$ is underestimated, and can partly compensate the overestimation of the previously mentioned lines.

Clearly, it appears that the models mainly based on the F10.7 proxy cannot be used for ionospheric calculations if the needed accuracy is better than $15 \%$. Moreover, this also shows that some solar proxies based on the integrated solar EUV flux cannot be used for assessing the solar variability in the EUV range. However, it is important to keep in mind that the uncertainties on the cross sections or on atmospheric models are often larger than $15 \%$ as mentioned in Cessateur et al. (2012a). In parallel to these solar studies, much more effort needs to be spent clarifying these last points.

\subsection{Effect on the atomic oxygen red line production}

In our study, we focus on the red line production, mainly because of its role in the interactions between molecular oxygen and the solar UV flux above $115 \mathrm{~nm}$. More specifically, at high altitudes the solar Lyman- $\alpha$ represents more than $25 \%$ of the red line production (cf. Fig. 5).

In comparison, the $\mathrm{O}$ I triplet at $130 \mathrm{~nm}$ represents less than $2 \%$. The rest of the red line production is mainly due to

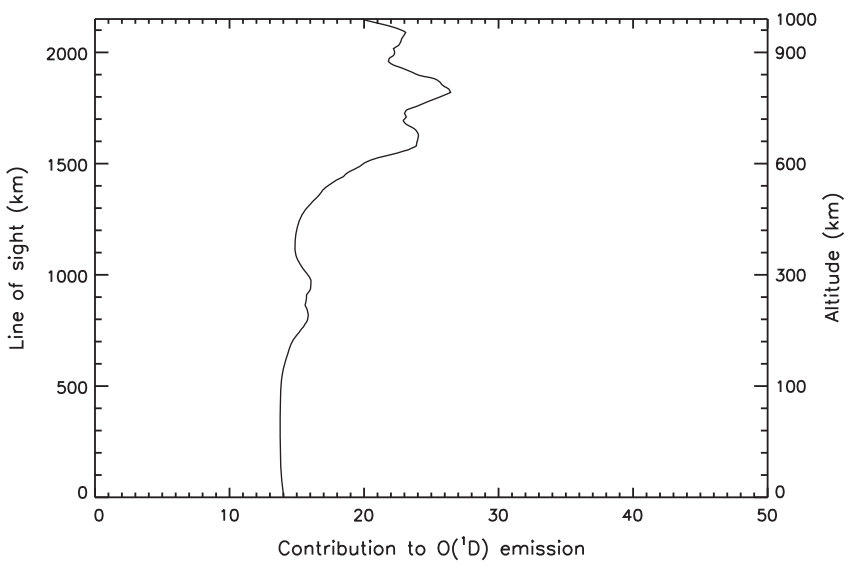

Fig. 5. Contribution to the red line emission from Lyman $\alpha$.

the continuum above $130 \mathrm{~nm}$. This result reveals how important it is to estimate carefully the irradiance intensity for specific solar lines, such as Lyman $\alpha$ in our case. In terms of atmospheric diagnostic, uncertainty on irradiance variability or even on the shape of one solar line can lead to quite biased derivation of atmospheric parameters like the precipitated electron flux or the atomic oxygen concentration. However, in the case of the transport calculation done with a transport code, the line profile is not critical, and only the integrated intensity is important.

\section{Example of Uranus: FUV continuum and solar reflection}

Recent HST (Hubble Space Telescope) observations of Uranus in the FUV allowed an Earth-based detection of an auroral event on November 29, 2011 (Lamy et al. 2012). Interpretations of the recorded spectra between 130 and $170 \mathrm{~nm}$ have been made, giving an estimation of the precipitating electron flux (Barthélemy et al. 2014) during the auroral event. The light recorded from Uranus by HST is composed of three main components: the solar reflected part, the airglow, and the $\mathrm{H}_{2}$ emission induced by energetic electron precipitation. In this study, the solar reflected component is by far the most important, and represents $\sim 90 \%$ of the signal as shown in Figure 6 . Thus any uncertainties in the solar flux will strongly modify the estimation of the other components of the signal. The concerned spectral region corresponds to the beginning of strong solar continuum added to important spectral lines. At these wavelengths, the total intensity variation over a solar cycle is around 15\% (Curdt \& Tian 2010).

The two last components of the Uranus spectra strongly depend on the solar UV flux both in the FUV and EUV while the solar reflected one depends only on the FUV part. In order to interpret these dominant contributions, Barthélemy et al. (2014) used albedo data from Yelle et al. (1989) deduced from Voyager data, and solar flux data from the TIMED-SEE instrument. They considered no uncertainties on the solar flux, and thus deduced both a new albedo of the planet in the studied spectral range and electron fluxes.

The conclusions from Barthélemy et al. (2014) are accurate only if the confidence in the solar UV fluxes is good. Since the solar reflected component represents $90 \%$ of the signal, the error on the solar flux becomes critical: an error of $1 \%$ on the flux can give rise to an error of at least $10 \%$ on the 


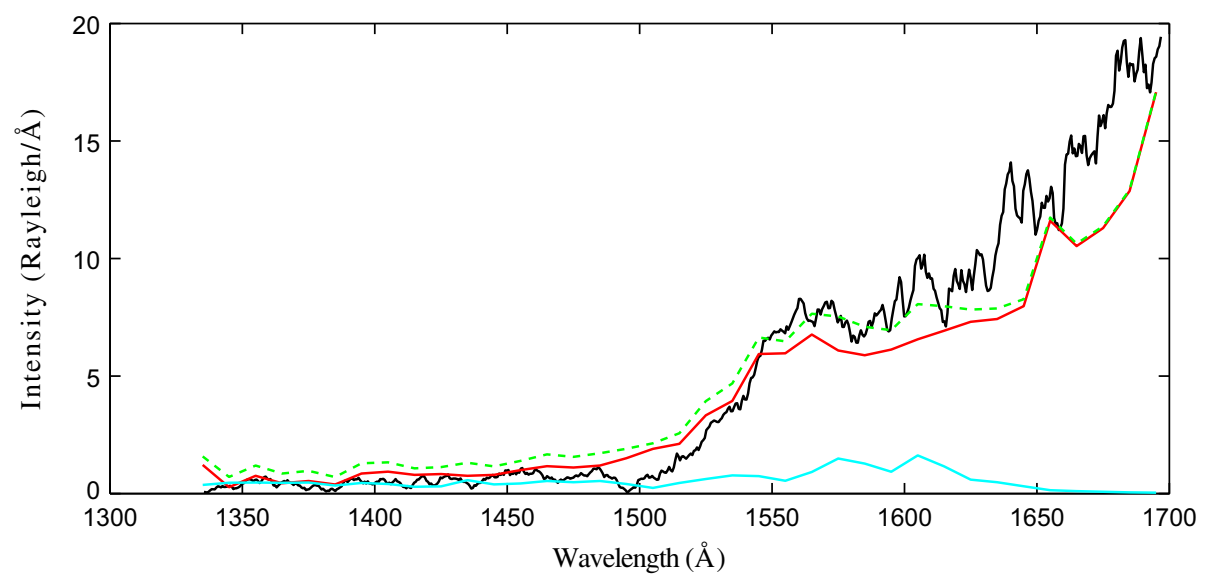

Fig. 6. Different contributions of the HST spectrum of Uranus, recorded November 29, 2011. The black line is the HST spectrum, the blue line is the modeled $\mathrm{H}_{2}$ spectrum, the red line represents the reflected spectrum, and the green asterisks are the sum of the two previous components. Figure adapted from Barthélemy et al. (2014).

estimation of the airglow and precipitation contributions, which contribute to planetary upper atmosphere and auroral processes. This error is in fact enhanced since the Uranus data are faint and thus noisy. If the solar absolute flux data are less accurate than a few percent, the absolute values deduced from this study are uncertain. This forces the question: Is the detection of the auroral event reported in Barthélemy et al. (2014) significant?

Studies of the spectral shape of the additional emissions could give interesting additional information. However, the error bars when looking at the spectral component are much larger than in the case of integrated intensity. In the case of this study, they are as large as the additional spectral signal, and the spectral shape is very uncertain: only the integrated values are really significant.

The albedo data time scale is much longer than the time scale of an auroral event. Despite the albedo data being uncertain, it is possible to consider them as constant on the time scale of one measurement campaign. ${ }^{2}$ Such is not the case with the solar flux. In Barthélemy et al. (2014), the auroral event is detected by an enhancement of $6 \%$ of the flux in the spectral region considered. During the period of the observation, the integrated solar flux varies up to about $1 \%$. This enhancement is significant only if short time scale variability of these instruments is much lower than the variation measured which is the case when averaging the data over few days. Thus the detection is significant, as stated in Barthélemy et al. (2014).

This point underscores the view that if the absolute values of the solar flux are important, the relative variation over a few days has to be much more accurate by at least one order of magnitude. Otherwise uncertainties would increase, and the interpretation of dayside data would be meaningless.

This particular case, namely where the reflected spectra of the planet are the major component of the detected emission, is one of the most unfavorable cases of all. However, such is the case for all the outer planets, including Mars, measurements taken from Earth's orbit. The jovian and saturnian cases are less critical since the auroral emissions are more intense but the martian auroras have only been detected by the SPICAM (Spectroscopy for Investigation of Characteristics of the Atmosphere of Mars) instrument on board Mars Express

\footnotetext{
${ }^{2}$ This is not the case for comparison between 2011 HST data and Voyager 2 data recorded in 1986 (Barthélemy et al. 2014).
}

(Bertaux et al. 2005). Attaining a detection of an aurora on Neptune from Earth's orbit with HST is a strong challenge, which will necessitate a very high precision on the solar flux. This shows that long-term measurements of the solar fluxes are mandatory since the models cannot reach accuracy of the order of a few percent. The stability of these instruments within a few days has to be better by at least one order of magnitude, as compared to the few percent mentioned previously.

It is also important to mention that uncertainties on the solar EUV part can also give rise to a bias in the dayglow and auroral intensity calculations. In Barthélemy et al. (2014), the Trans* code family is used to calculate the dayglow and the auroral components. As for Ganymede, the EUV part is important, and is used as an input for the calculation of the processes in the upper atmosphere and their emissions. It appears to be less critical, but the uncertainties on instrumental solar EUV measurements are much larger compared to those in the FUV spectral range (30\% versus $2 \%-3 \%)$ as described in Woods et al. (2005) and Rottman (2005); the variability is also more important (40\%-100\% versus $2 \%-3 \%$ over the solar 11 -year cycle). The uncertainty on the solar EUV flux thus cannot be totally negligible even if the uncertainties on the cross sections are often more important (often up to 50\%).

\section{Optically thick cases: example of the planetary H-Lyman $\alpha$ emissions and the 0 I $130 \mathrm{~nm}$ triplet}

In planetary atmospheres, some spectral lines are scattered by the upper atmosphere such as O I $130 \mathrm{~nm}$ or H-Lyman $\alpha$. These lines are optically thick in such atmospheres and resonant with the solar spectrum. The intensity and line shape of such emissions can also give information on the upper atmosphere thermodynamics (Barthélemy et al. 2014) or composition (Chaufray et al. 2009). Since Lyman $\alpha$ is by far the most intense line in the FUV solar spectrum, this line, alone, can significantly modify the atmospheric structure and thermodynamics. Recent models showed that the Lyman $\alpha$ cooling can significantly modify the temperature of the upper atmosphere of hot Jupiters (Menager et al. 2013).

In these cases, the problem is to know precisely the full solar disk intensity and the profile of one particular emission line. H-Lyman $\alpha$ has been extensively studied and measured 
especially the line profile and intensity by instruments like OSO8 or SUMER (Curdt et al. 2008 and references herein). Even if it seems to be quite symmetric in allowing a double Gaussian shape, some variations over the solar cycle occur (Lemaire et al. 2005). Since the SUMER instrument failure, no high resolution instrument is recording any Lyman $\alpha$ line profiles. SORCE and LYRA both have measured the Lyman $\alpha$ flux. However, LYRA is not well calibrated in terms of absolute flux, and has no spectral resolution (Dominique et al. 2013), and SORCE presents a small spectral resolution of about $1 \mathrm{~nm}$ at these wavelengths. Thus they cannot provide information on the line profile.

In terms of planetary radiative transfer, and hence upper atmosphere diagnostics, this full disk spectral line profile is the relevant input. For most of the simulations a double Gaussian intensity is used as in Menager et al. (2010). If we consider that the upper atmospheric temperature is much lower than the emission temperature of the stellar line (around 30,000 K for the center of the line and between 4000 and $7000 \mathrm{~K}$ for the line wings; Vernazza et al. 1981), only the center of the line is scattered by the planetary atmosphere. Regarding this, we point out that a small change in the line shape or an asymmetry as shown in Tian et al. (2009) can change the intensity effectively scattered by the planet, and if not taken into account, leads to bias in the derived atmospheric parameters. It is in particular critical when the H-Lyman $\alpha$ self-reversal becomes more important since it will significantly change the intensity at the center of the line. Since only a thin part of the center of the line is absorbed or scattered when the atmosphere is cold, it is more critical for cold exospheres like in Mars or Venus where the planetary line width is much smaller.

As an example we tested the sensitivity of Jupiter's H-Lyman $\alpha$ planetary line to the solar line profile. We used the radiative transfer code mentioned in Barthélemy et al. (2005) and Menager et al. (2010). In these studies, the Lyman $\alpha$ line is used as a diagnostic tool for the upper atmospheres. The same double gaussian lines were used as in these previous articles i.e. an offset of each peak equal to $0.022 \mathrm{~nm}$ for the line center and a width of each line equal to $0.0216 \mathrm{~nm}$. The line of sight is at the nadir in the equatorial plane with a solar longitude of $20^{\circ}$. We tested the sensitivity of the total intensity regarding the width of each line of the double gaussian shape using a constant integrated flux. The enhancement of $15 \%$ to the line width infers an enhancement of $5 \%$ of the total intensity. On the contrary, an enhancement of $15 \%$ of the offset between the two gaussian peaks leads to a diminution of the planetary emitted flux but it is much more important than in the previous case representing $20 \%$ of the total intensity. The sensitivity to the line profile means that an uncertainty on the line shape can lead to errors in the calculated intensity and thus on the deduced conclusions. For example, Barthélemy et al. (2005) deduced the vibrational temperatures to be $40 \%$ larger than the kinetic ones in the jovian upper atmosphere from absorption of a small part of the line due to $\mathrm{H}_{2}$. A change of $15 \%$ on the line width will enhance these absorptions, and can lead to the conclusion that the vibrational temperatures are around $60 \%$ larger than the kinetic ones.

In the previous example, only atmospheric lines resonant with solar lines are considered. This is mainly the case with H-Lyman lines and O I $130 \mathrm{~nm}$ triplet. This means that a reduced number of EUV/FUV solar emission are concerned. However, the dayside emission of the upper atmosphere of a planet can also be induced by fluorescence of intense solar lines on atmospheric molecular lines when wavelength coincidences exist. For example, this is the case with a number of solar lines between $80 \mathrm{~nm}$ and $120 \mathrm{~nm}$ with $\mathrm{H}_{2}$ Lyman and Werner bands for the case of giant planets. This can also be the situation with the fourth positive band of $\mathrm{CO}\left(\mathrm{A}^{1} \Pi-\mathrm{X}^{1} \Sigma^{+}\right)$ for Mars (Feldman et al. 2000). The fluorescence on $\mathrm{H}_{2}$ has been for example calculated in the case of Jupiter by Liu \& Dalgarno (1996), and gives rise to an airglow emission, which can represent the half of the total $\mathrm{H}_{2}$ airglow. It is also the case for Uranus where it represents more than $20 \%$ of the airglow (Barthélemy et al. 2014). Since these fluorescences are due to coincidences between solar atomic or ionic lines and thin planetary molecular lines, the solar line profile is really important. Unfortunately, except for the H-Lyman $\alpha$ and $\beta$ lines, the profiles are poorly known. Liu \& Dalgarno (1996) and Barthélemy et al. (2014) used a simple gaussian shape with a standard deviation of $0.1 \AA$ to model all the other solar line widths. As an example, we choose the N III solar line at $99.155 \mathrm{~nm}$, which shows a close coincidence with the 9-0 $\mathrm{R}(1)$ with a wavelength difference of $0.17 \AA$. With a width of $0.1 \AA$ the fluorescence will be nonnegligible. However, if the width is twice smaller $0.05 \AA$, the intensity of the fluorescence is divided by more than 200 , and becomes totally negligible. This shows that the solar line profile is really critical in this case since the fluorescence needs very close coincidence. Until present date, the spectrometer with the highest resolution was SUMER with a resolution of $\sim 0.045 \AA$, and it was not able to measure full disk emission lines. In order to measure line shape with width of the order of $0.1 \AA$ a future instrument will have to reach a spectral resolution of $0.01 \AA$ (considering that 10 points is a minimum to get the line profile). Currently in the FUV, only HST STIS (Space Telescope Imaging Spectrograph) with the echelle grating E140L can reach such a resolution. FUSE (Far Ultraviolet Spectroscopic Explorer) also had a resolution of $0.02 \AA$ in the EUV (not far from this requirement), which is not the case for EXCEED (Extreme Ultraviolet Spectroscope for Exospheric Dynamics) where it only reaches $0.3-0.5 \mathrm{~nm}$.

\section{Stellar UV flux in the case of exoplanets}

Since the discovery of a large panel of exoplanets (940 at the end of August 2013; www.exoplanet.eu) and the detection by transit absorption of two atmospheres (Vidal-Madjar et al. 2003; Lecavelier Des Etangs et al. 2010), the question of a diagnostic of their upper atmospheres using the thermospheric emissions is open (Menager et al. 2013). As stated in Menager et al. (2013) for exoplanets and in Section 5 of this paper for giant planets, the problem of the intensity and shape of the Lyman $\alpha$ line is critical to model the emission coming from the planet considering the fact that it represents the main part of the planetary emission. It is also important to notice that the exosphere or hydrogen corona of these planets can reach very high temperatures above $10^{4} \mathrm{~K}$, and thus enhance the planetary line width.

In order to explore possible future auroral or dayglow diagnostic of exoplanets, the calculations in Menager et al. (2013) also used the family of Trans* code simulations, calculating the transport of the electrons through the atmosphere. This simulation thus needs the EUV and FUV flux (the stellar wind and the magnetospheric interactions also) of a large panel of stars hosting planets. This faces several problems: since FUSE (decommissioned in Oct. 2007), no EUV instrument except EXCEED is flying. In the FUV, only HST can give information 
from exoplanetary systems. In addition, large uncertainties remain on the line profile emitted by the stars since it is largely absorbed by the interstellar medium.

However some recent studies give important information on these EUV/FUV fluxes. Sanz-Forcada et al. (2011) tried to estimate the EUV flux from the $\mathrm{X}$ ray flux for a panel of stars with planets. Linsky et al. (2013) reconstructed the Lyman $\alpha$ flux for a panel of nearby stars from M5 V to F5 V spectral types with estimated uncertainties up to $30 \%$. France et al. (2013) reconstructed the radiative UV environment around $\mathrm{M}$ dwarf stars hosting planets with high UV fluxes. Clearly, these efforts must be continued, and they underline the need for future UV space telescopes (Gómez de Castro et al. 2014). In the EUV range between $55 \mathrm{~nm}$ and $145 \mathrm{~nm}$, the satellite EXCEED built by JAXA (Japan Aerospace Exploration Agency) can be a useful tool to get some stellar spectra even if it has not been designed for this.

\section{Conclusion}

From the study of three examples, we have shown the importance of an accurate knowledge of solar and stellar UV fluxes for planetary upper atmospheres studies. The most critical case appears when a strong reflected component of the solar light is mixed with the atmospheric data. As shown, the needed precision is of the order of few percent with a time stability within few days better than $1 \%$. The other cases are less critical. However due to the strong variability of the UV fluxes, which is really important for space weather purposes, they also need good accuracies and stabilities. This study also underlines the need for long-term measurements with very high resolution line profiles and large spectral coverage. Considering the recent discoveries of exoplanets, it appears that a strong effort for the study of a large panel of nearby stars is needed. Approximately 100 known planets are at less than $20 \mathrm{pc}$ from the solar system. Future UV space observatories will be needed for this extensive analysis.

Acknowledgements. The authors thank Christopher Parkinson and Wilnelia Adams for helping to improve the manuscript especially the language. The editor thanks Thomas E. Cravens and one anonymous referee for their assistance in evaluating this paper.

\section{References}

Barthélemy, M., L. Lamy, H. Menager, M. Schulik, D. Bernard, H. Abgrall, E. Roueff, G. Cessateur, R. Prange, and J. Lilensten. Dayglow and auroral emissions of Uranus in $\mathrm{H}_{2}$ FUV bands, Icarus, 239, 160-167, 2014, DOI: 10.1016/j.icarus.2014.05.035.

Barthélemy, M., J. Lilensten, and C. Parkinson. $\mathrm{H}_{2}$ vibrational temperatures in the upper atmosphere of Jupiter, $A \& A, \mathbf{4 3 7}$, 329-331, 2005, DOI: 10.1051/0004-6361:20040257.

BenMoussa, A., S. Gissot, U. Schühle, G. Del Zanna, F. Auchère, et al. On-orbit degradation of solar instruments, Solar Phys., 288, 389-434, 2013, DOI: 10.1007/s11207-013-0290-z.

Bertaux, J.-L., F. Leblanc, O. Witasse, E. Quemerais, J. Lilensten, S.A. Stern, B. Sandel, and O. Korablev. Discovery of an aurora on Mars, Nature, 435, 790-794, 2005, DOI: 10.1038/nature03603.

Cessateur, G., Reconstruction du spectre UV solaire en vue de la caractérisation des environnements plantaires. Ph.D. thesis, Université d'Orléans (in French), 2011.

Cessateur, G., T. Dudok de Wit, M. Kretzschmar, J. Lilensten, J. Hochedez, and M. Snow. Monitoring the solar UV irradiance spectrum from the observation of a few passbands, $A \& A, \mathbf{5 2 8}$, A68, 2011, DOI: 10.1051/0004-6361/201015903.
Cessateur, G., J. Lilensten, M. Barthélémy, T. Dudok de Wit, C. Simon Wedlund, G. Gronoff, H. Ménager, and M. Kretzschmar. Photoabsorption in Ganymede's atmosphere. Icarus, 218, 308-319, 2012a, DOI: 10.1016/j.icarus.2011.11.025.

Cessateur, G., J. Lilensten, T. Dudok de Wit, A. BenMoussa, and M. Kretzschmar. New observation strategies for the solar UV spectral irradiance. J. Space Weather Space Clim., 2, A16, 2012b, DOI: $10.1051 / \mathrm{swsc} / 2012016$.

Chaufray, J.Y., F. Leblanc, E. Quémerais, and J.L. Bertaux. Martian oxygen density at the exobase deduced from O I 130.4-nm observations by Spectroscopy for the investigation of the characteristics of the atmosphere of Mars on Mars Express. J. Geophys. Res. (Planets), 114, E02006, 2009, DOI: $10.1029 / 2008 J E 003130$.

Crane, P.C., L.E. Floyd, J.W. Cook, L.C. Herring, E.H. Avrett, and D.K. Prinz. The center-to-limb behavior of solar active regions at ultraviolet wavelengths. $A \& A, \mathbf{4 1 9}, 735-746,2004$, DOI: 10.1051/0004-6361:20040012.

Curdt, W., and H. Tian. Hydrogen Lyman Emission through the Solar Cycle. In: S.R., Cranmer, J.T. Hoeksema, and J.L. Kohl, Editors, SOHO-23: understanding a peculiar solar minimum, vol. 428 of Astronomical Society of the Pacific Conference Series, $81,2010$.

Curdt, W., H. Tian, L. Teriaca, U. Schühle, and P. Lemaire. The Ly- $\alpha$ profile and center-to-limb variation of the quiet Sun. $A \& A, \mathbf{4 9 2}$, L9-L12, 2008, DOI: 10.1051/0004-6361:200810868.

Domingo, V., I. Ermolli, P. Fox, C. Fröhlich, M. Haberreiter, et al. Solar surface magnetism and irradiance on time scales from days to the 11-year cycle. Space Sci. Rev., 145, 337-380, 2009, DOI: $10.1007 / \mathrm{s} 11214-009-9562-1$

Dominique, M., J.-F. Hochedez, W. Schmutz, I.E. Dammasch, A.I. Shapiro, M. Kretzschmar, A.N. Zhukov, D. Gillotay, Y. Stockman, and A. BenMoussa. The LYRA instrument onboard PROBA2: description and in-flight performance. Solar Phys., 286, 21-42, 2013, DOI: 10.1007/s11207-013-0252-5.

Dudok de Wit, T., M. Kretzschmar, J. Lilensten, and T. Woods. Finding the best proxies for the solar UV irradiance. Geophys. Res. Lett., 36, 10107, 2009, DOI: 10.1029/2009GL037825.

Feldman, P.D., E.B. Burgh, S.T. Durrance, and A.F. Davidsen. Far-ultraviolet spectroscopy of Venus and Mars at $4 \AA$ resolution with the Hopkins Ultraviolet Telescope on Astro-2. Astrophys. J., 538, 395-400, 2000, DOI: 10.1086/309125.

Floyd, L., J. Newmark, J. Cook, L. Herring, and D. McMullin. Solar EUV and UV spectral irradiances and solar indices. J. Atmos. Sol. Terr. Phys., 67, 3-15, 2005, DOI: 10.1016/j.jastp.2004.07.013.

France, K., C.S. Froning, J.L. Linsky, A. Roberge, J.T. Stocke, et al. The ultraviolet radiation environment around $\mathrm{M}$ dwarf exoplanet host stars. Astrophys. J., 763, 149, 2013, DOI: 10.1088/0004-637X/763/2/149.

Galand, M., L. Moore, I. Mueller-Wodarg, M. Mendillo, and S. Miller. Response of Saturn's auroral ionosphere to electron precipitation: electron density, electron temperature, and electrical conductivity. J. Geophys. Res. (Space Physics), 116, A09306, 2011, DOI: 10.1029/2010JA016412.

Gómez de Castro, A.I., T. Appourchaux, M.A. Barstow, M. Barthelemy, F. Baudin, et al. Building galaxies, stars, planets and the ingredients for life between the stars. The science behind the European Ultraviolet-Visible Observatory. Astrophys. Space Sci., 354 (1), 229, 2014, DOI: 10.1007/s10509-014-1942-7.

Grodent, D., J.H. Waite Jr., and J.-C. Gerard. A self-consistent model of the Jovian auroral thermal structure. J. Geophys. Res., 106, 12933-12952, 2001, DOI: 10.1029/2000JA900129.

Gronoff, G., J. Lilensten, L. Desorgher, and E. Flückiger. Ionization processes in the atmosphere of Titan. I. Ionization in the whole atmosphere. $A \& A, \mathbf{5 0 6}, 955-964,2009$ a, DOI: $10.1051 / 0004-636 / 2009123711$.

Gronoff, G., J. Lilensten, and R. Modolo. Ionization processes in the atmosphere of Titan. II. Electron precipitation along magnetic field lines. $A \& A, \mathbf{5 0 6}, 965-970,2009 \mathrm{~b}$,

DOI: $10.1051 / 0004-6361 / 200912125$. 
Gronoff, G., J. Lilensten, C. Simon, M. Barthélemy, F. Leblanc, and $\mathrm{O}$. Dutuit. Modelling the Venusian airglow. $A \& A, \mathbf{4 8 2}$, 1015-1029, 2008, DOI: 10.1051/0004-6361:20077503.

Gronoff, G., C. Simon Wedlund, C.J. Mertens, M. Barthélemy, R.J. Lillis, and O. Witasse. Computing uncertainties in ionosphereairglow models: II. The Martian airglow. J. Geophys. Res. (Space Physics), 117, A05309, 2012, DOI: 10.1029/2011JA017308.

Haigh, J.D., A.R. Winning, R. Toumi, and J.W. Harder. An influence of solar spectral variations on radiative forcing of climate. Nature, 467, 696-699, 2010, DOI: 10.1038/nature09426.

Hinteregger, H.E. Representations of solar EUV fluxes for aeronomical applications. Adv. Space Res., 1, 39-52, 1981, DOI: 10.1016/0273-1177(81)90416-6.

Lamy, L., R. Prangé, K.C. Hansen, J.T. Clarke, P. Zarka, et al. Earthbased detection of Uranus' aurorae. Geophys. Res. Lett., 39, L07105, 2012, DOI: 10.1029/2012GL051312.

Lean, J.L., H.P. Warren, J.T. Mariska, and J. Bishop. A new model of solar EUV irradiance variability 2. Comparisons with empirical models and observations and implications for space weather. J. Geophys. Res. (Space Phys.), 108, 1059, 2003, DOI: 10.1029/2001JA009238.

Lean, J.L., T.N. Woods, F.G. Eparvier, R.R. Meier, D.J. Strickland, J.T. Correira, and J.S. Evans. Solar extreme ultraviolet irradiance: present, past, and future. J. Geophys. Res. (Space Phys.), 116, A01102, 2011, DOI: 10.1029/2010JA015901.

Lecavelier Des Etangs, A., D. Ehrenreich, A. Vidal-Madjar, G.E. Ballester, J.-M. Désert, R. Ferlet, G. Hébrard, D.K. Sing, K.-O. Tchakoumegni, and S. Udry. Evaporation of the planet HD 189733b observed in H I Lyman- $\alpha$. A\&A, 514, A72, 2010, DOI: $10.1051 / 00046361 / 200913347$.

Lemaire, P., C. Emerich, J.-C. Vial, W. Curdt, U. Schühle, and K. Wilhelm. Variation of the full Sun hydrogen Lyman profiles through solar cycle 23. Adv. Space Res., 35, 384-387, 2005, DOI: $10.1016 /$ j.asr.2004.11.004.

Lilensten, J., and P.L. Blelly. The TEC and F2 parameters as tracers of the ionosphere and thermosphere. J. Atmos. Sol. Terr. Phys, 64, 775-793, 2002, DOI: 10.1016/S1364-6826(02)00079-2.

Lilensten, J., T. Dudokdewit, M. Kretzschmar, P. Amblard, S. Moussaoui, J. Aboudarham, and F. Auchère. Review on the solar spectral variability in the EUV for space weather purposes. Ann. Geophys., 26, 269-279, 2008.

Lilensten, J., C. Simon, O. Witasse, O. Dutuit, R. Thissen, and C. Alcaraz. A fast computation of the diurnal secondary ion production in the ionosphere of Titan. Icarus, 174, 285-288, 2005a, DOI: 10.1016/j.icarus.2004.12.002.

Lilensten, J., O. Witasse, C. Simon, H. Soldi-Lose, O. Dutuit, R. Thissen, and C. Alcaraz. Prediction of a $\mathrm{N}_{2}^{++}$layer in the upper atmosphere of Titan. Geophys. Res. Lett., 32, L03, 203, 2005b, DOI: 10.1029/2004GL021432.

Linsky, J.L., K. France, and T. Ayres. Computing intrinsic LY $\alpha$ fluxes of F5 V to M5 V Stars. Astrophys. J., 766, 69, 2013, DOI: $10.1088 / 0004-637 \mathrm{X} / 766 / 2 / 69$.

Liu, W., and A. Dalgarno. The ultraviolet spectrum of the Jovian dayglow. Astrophys. J., 462, 502, 1996, DOI: 10.1086/177168.

Marconi, M.L. Akinetic model of Ganymede's atmosphere. Icarus, 190, 155-174, 2007, DOI: 10.1016/j.icarus.2007.02.016.

Menager, H., M. Barthélemy, T. Koskinen, J. Lilensten, D. Ehrenreich, and C.D. Parkinson. Calculation of the H Lyman $\alpha$ emission of the hot Jupiters HD 209458b and HD 189733b. Icarus, 226, 1709-1718, 2013,

DOI: $10.1016 /$ j.icarus.2013.02.028.

Menager, H., M. Barthélemy, and J. Lilensten. H Lyman $\alpha$ line in Jovian aurorae: electron transport and radiative transfer coupled modelling. $A \& A, \mathbf{5 0 9}$, A56, 2010 ,

DOI: $10.1051 / 0004-6361 / 200912952$.
Mikhailov, A., A. Belehaki, L. Perrone, B. Zolesi, and I. Tsagouri. Retrieval of thermospheric parameters from routine ionospheric observations: assessment of methods performance at midlatitudes daytime hours. J. Space Weather Space Clim., 2, A03, 2012.

Richards, P.G., J.A. Fennelly, and D.G. Torr. EUVAC: a solar EUV flux model for aeronomic calculations. J. Geophys. Res., 99, 8981-8992, 1994, DOI: 10.1029/94JA00518.

Richards, P.G., T.N. Woods, and W.K. Peterson. HEUVAC: a new high resolution solar EUV proxy model. Advances in Space Research, 37, 315-322, 2006, DOI: 10.1016/j.asr.2005.06.031.

Rottman, G. The SORCE mission. Solar Phys., 230, 7-25, 2005, DOI: $10.1007 / \mathrm{s} 11207-005-8112-6$.

Sanz-Forcada, J., G. Micela, I. Ribas, A.M.T. Pollock, C. Eiroa, A. Velasco, E. Solano, and D. García-Álvarez. Estimation of the XUV radiation onto close planets and their evaporation. $A \& A$, 532, A6, 2011, DOI: 10.1051/0004-6361/201116594.

Shematovich, V.I., D.V. Bisikalo, J.-C. Gérard, C. Cox, S.W. Bougher, and F. Leblanc. Monte Carlo model of electron transport forthe calculation of Mars dayglow emissions. J. Geophys. Res. (Planets), 113, E02011, 2008, DOI: 10.1029/2007JE002938.

Simon, C., O. Witasse, F. Leblanc, G. Gronoff, and J. Bertaux. Dayglow on Mars: Kinetic modelling with SPICAM UV limb data. Planet. Space Sci., 57, 1008-1021, 2009, DOI: $10.1016 /$ j.pss.2008.08.012.

Tian, H., W. Curdt, E. Marsch, and U. Schühle. Hydrogen Lyman- $\alpha$ and Lyman- $\beta$ spectral radiance profiles in the quiet Sun. $A \& A$, 504, 239-248, 2009, DOI: 10.1051/0004-6361/200811445.

Tobiska, W.K., T. Woods, F. Eparvier, R. Viereck, L. Floyd, D. Bouwer, G. Rottman, and O.R. White. The SOLAR2000 empirical solar irradiance model and forecast tool. J. Atmos. Sol. Terr. Phys., 62, 1233-1250, 2000,

DOI: $10.1016 / \mathrm{S} 1364-6826(00) 00070-5$.

Vernazza, J.E., E.H. Avrett, and R. Loeser. Structure of the solar chromosphere. III - Models of the EUV brightness components of the quiet-sun. Astrophys. J. Suppl. Ser., 45, 635-725, 1981, DOI: $10.1086 / 190731$.

Vidal-Madjar, A., A. Lecavelier des Etangs, J.-M. Désert, G.E. Ballester, R. Ferlet, G. Hébrard, and M. Mayor. An extended upper atmosphere around the extrasolar planet HD209458b. Nature, 422, 143-146, 2003, DOI: 10.1038/nature01448.

Witasse, O., O. Dutuit, J. Lilensten, R. Thissen, and J. Zabka, et al. Prediction of a $\mathrm{CO}_{2}^{2+}$ layer in the atmosphere of Mars. Geophys. Res. Lett., 29 (8), 2002, 12-63, DOI: 10.1029/2002GL014781.

Witasse, O., O. Dutuit, J. Lilensten, R. Thissen, and J. Zabka, et al. Correction to "Prediction of a $\mathrm{CO}_{2}^{2+}$ layer in the atmosphere of Mars". Geophys. Res. Lett., 30 (7), 1360, 2003, DOI: $10.1029 / 2003$ GL017007.

Woods, T.N., P.C. Chamberlin, W.K. Peterson, R.R. Meier, and P.G. Richards, et al. XUV Photometer System (XPS): improved solar irradiance algorithm using CHIANTI Spectral Models. Solar Phys., 250, 235-267, 2008, DOI: 10.1007/s11207-008-9196-6.

Woods, T.N., F.G. Eparvier, S.M. Bailey, P.C. Chamberlin, J. Lean, G.J. Rottman, S.C. Solomon, W.K. Tobiska, and D.L. Woodraska. Solar EUV Experiment (SEE): mission overview and first results. J. Geophys. Res. (Space Phys.), 110, A01312, 2005, DOI: 10.1029/2004JA010765.

Yelle, R.V., J.C. Mcconnell, and D.F. Strobel. The far ultraviolet reflection spectrum of Uranus - Results from the Voyager encounter. Icarus, 77, 439-456, 1989, DOI: $10.1016 / 0019-1035(89) 90098-5$.

Cite this article as: Barthelemy, M. and G. Cessateur. Sensitivity of upper atmospheric emissions calculations to solar/stellar UV flux. J. Space Weather Space Clim., 4, A35, 2014, DOI: 10.1051/swsc/2014033. 